\title{
Enslaving random fluctuations in nonequlibrium systems
}

\author{
Mangal C. Mahato, T. P. Pareek and A. M. Jayannavar \\ Institute of Physics, Sachivalaya Marg, Bhubaneswar-751005, INDIA.
}

\begin{abstract}
Several physical models have recently been proposed to obtain unidirectional motion of an overdamped Brownian particle in a periodic potential system. The asymmetric ratchetlike form of the periodic potential and the presence of correlated nonequilibrium fluctuating forces are considered essential to obtain such a macroscopic motion in homogeneous systems. In the present work, instead, inhomogeneous systems are considered, wherein the friction coefficient and/or temperature could vary in space. We show that unidirectional motion can be obtained even in a symmetric nonratchetlike periodic potential system in the presence of white noise fluctuations. We consider four different cases of system inhomogeneity We argue that all these different models work under the same basic principle of alteration of relative stability of otherwise locally stable states in the presence of temperature inhomogeneity.
\end{abstract}

PACS No.:05.40.+j, 82.20.Mj 


\section{Introduction}

There has been much discussion recently[1-10] on how to model a physical system that could extract work out of random fluctuations without having to apply directly an obvious biased force taking, in fact, cue from —- or perhaps yearning to explain — an experimentally observed phenomena[11] of predominantly unidirectional motion of macromolecules (biological motors) along microtubules. We reason in the present work that system inhomogeneity may provide a clear and unifying framework to approach the problem of macroscopic motion under discussion. Macroscopic unidirectional motion of a particle is not possible thermodynamically in the prsence of equilibrium fluctuations. However, such a motion can be obtained in an nonequilibrium situation where the principle of detailed balance does not hold. The existing popular models, [1-10] currently in the literature, mostly take the fluctuations to be nonequilibrium, that is, consider nonwhite or at least nonguassian-white (colored) noise together with a ratchetlike periodic system potential to aid asymmetric motion of an overdamped Brownian particle.

The ratchetlike periodic system potentials, $V(q)$, obviously violate parity $V(q) \neq V(-q)$. For such a ratchetlike potential one can readily calculate steady current flow $J(F)$ of a Brownian particle in the presence of an external field $F$. It turns out that $J(F)$ is not an odd function of $F$ and, in general, $J(F) \neq-J(-F)$. In other words, reversal of the external force may not lead to a reversed current of the same magnitude in sharp contrast to the case of a nonratchetlike (symmetric) periodic potential system where $J(F)=-J(-F)$ follows. From this general observation, in a ratchetlike potential, it can be easily concluded that on application of a zero time averaged periodic field, say $F=F_{0} \sin \omega t$, one can obtain net unidirectional current. Of course, the direction and magnitude of the average velocity may depend, in a 
as on the external field parameters, $F_{0}$ and $\omega$. A careful tuning of the relevant parameters may even result in the reversal of the macroscopic current [2]. This is the basic physics behind some of the physical models used to obtain current rectification in a periodic potential system. There are models, however, that do not use oscillating external fields. Instead, colored noise of zero average strength--dichotomous, Ornstein-Uhlenbeck, Kangaroo processes,..., [3-5]— is used to drive the Brownian particle to obtain macroscopic motion in a ratchetlike potential system. There are further interesting models where the potential barriers themselves are allowed to fluctuate, for instance, with finite time correlations between two states under the influence of a noise source. An example being an overdamped Brownian particle subjected to a ratchetlike periodic potential, where the ratchetlike saw-tooth potential is switched on to its full strength for time $\tau_{\text {on }}$ during which the Brownian particle slides down the potential slope to the bottom of the potential trench. At the end of $\tau_{o n}$, the system is put in the other (off) state during which the potential is set equal to a constant (say $=0)$ for an interval $\tau_{\text {off }}$ and the particle executes force-free diffusive motion. At the end of $\tau_{\text {off }}$ the system is put back in the on state for interval $\tau_{o n}$. This process of flipping of states is repeated ad-infinitum. If $\tau_{\text {off }}$ is adjusted in such a way that by the end of $\tau_{\text {off }}$ the diffusive motion just takes the particle out of the (now nonexistent) potential trench in the steeper slope direction (smaller distance) of the saw-tooth potential but fails to do so in the gentler slope direction (larger distance), the immediate next on interval will take the particle to the adjacent trench minimum in the steeper slope side of the saw-tooth potential. Repititon of such sequential flippings of states for a large number of times leads to a net unidirectional macroscopic current of the Brownian particle. It should be noted that a symmetrical nonratchetlike potential would, instead, have yielded symmetrical excursions of the Brownian particle and, hence, no net unidirectional 
supplied with the required energy externally to flip the system between the two states keeping the interval $\tau_{o n}$ and $\tau_{\text {off }}$ fixed. There is a lot of freedom to play around with the parameters $\tau_{o n}, \tau_{o f f}$, the parameters of the saw tooth potential, and the thermal (Brownian) noise strength. And a judicious tuning of these parameters could even result in the reversal of the current. The flipping process could, however, be effected also by a finite time-correlated fluctuating dichotomous force. It should be noted that the former flipping proceses of definite $\tau_{o n}$ and $\tau_{\text {off }}$ have been practically exploited in the particle separation techniques, whereas the latter fluctuating flipping time process has some appeal to natural processes.

The diversity of the models just does not end here. There has been attempts too to obtain macroscopic current with Gaussian white noise under nonratchetlike symmetric periodic potential field as well but subjected to temporally asymmetric periodic external fields [8-10]. We do not attempt here, however, to give a review of the models considered. We present, in the following, a framework to obtain macroscopic motion in an inhomogeneous system with space dependent friction coefficient and nonuniform temperature fields restricting ourselves to periodic potential systems.

In all the models just mentioned [1-10] (The list is not exhaustive.) the system was taken to be homogeneous as far as the question of diffusivity was concerned. However, in some of the works, earlier to the ones eluded to so far, the nonuniformity of the diffusion constant of the system was considered to yield macroscopic transport [12-13 ]. The diffusion coefficient could be space dependent or state dependent and so the system may dissipate energy during its time evolution differently at different space points. Unlike homogeneous systems, however, the physics of inhomogeneous systems has not been free from controversies, such as, whether 
the equation

$$
\frac{\partial P}{\partial t}=\frac{\partial^{2}}{\partial q^{2}} D(q) P
$$

or

$$
\frac{\partial P}{\partial t}=\frac{\partial}{\partial q} D(q) \frac{\partial P}{\partial q}
$$

should be the correct form of diffusion equation. Nevertheless, such controversies apart, Büttiker [12] and also van Kampen [13] have shown that one can expect macroscopic transport of a Brownian particle in a periodic potential field when the diffusion coefficient is also periodic with the same periodicity but shifted by a phase difference other than 0 and $\pi$ with respect to the periodic potential field. It should be noted that the potential field is not required to be ratchetlike. The system is rendered nonequilibrium by diffusion coefficient inhomogeneity in the system and the "stationary state" of the system is no longer goverened by the usual Boltzmann factor.

In reference [12], the source of inhomogeneity is not dwelt into. However, there exist literature, that discuss at length the thermodynamic origin of the possible inhomogeneity of the diffusion coefficient [14]. The inhomogeneity could arise from the nonuniformity of the friction coefficient and/or from the nonuniformity of the temperature of the macroscopic sample. The friction coefficient variation changes the rate of relaxation of the system, whereas the temperature variation even alters the relative stability of the otherwise locally stable states. The idea of the change of relative stability of states due to temperature inhomogeneity was advanced by Landauer [14]. This idea has already been employed to show the possibility to generate 
important to understand the contributions of the friction coefficient inhomogeneity and the nonuniformity of temperature separately for, as stated earlier, they influence the transport properties of the system in two different ways. Moreover, to clarify the physical meaning of various terms, in the theory, one needs to go beyond the phenomenological description.

In reference [15], a microscopic treatment is given for the derivation of the macroscopic equations of motion in an inhomogeneous medium (space dependent friction coefficient and spatially nonuniform temperature) starting from a microscopic Hamiltonian of the system in contact with (phonon) heat bath(s). Moreover, a proper overdamped limit of the Langevin equation of motion in such an inhomogeneous medium is derived. A correct form of the corresponding Fokker-Planck equation is obtained and it is explicitly shown that neither of the two forms of the diffusion equation mentioned above [eq.(1)] is correct. From this macroscopic equation of motion one obtains an expression for the average current which depends on the details of the potential field and the inhomogeneities of the system. This microscopic treatment, however, helps in understanding the functioning of the recently proposed model of Maxwell's demon type information engine [16].

The Maxwell's demon type information engine in which the particle is coupled to two thermal baths at temperatures $T$ and $\bar{T}$ was investigated in reference $[16,17]$. It has been shown that the engine extracts work out of a nonequlibrium environment $(T \neq \bar{T})$ by rectifying internal (white noise) fluctuations and the Brownian particle, thus, acquires a nonzero macroscopic velocity. A correct analytical expression for the average macroscopic velocity of the Brownian particle is derived and it is shown[17] that in an approximate range of physical parameters the average velocity so acquired is similar to that of a particle evolving in an inhomogeneous medium(see below). 
the space dependence of the friction coefficient $\eta(q)$ or that of the temperature $T(q)$ or because of both. In physics such inhomogeneous systems are not so uncommon. Examples of such systems include systems with semiconductor junctions and a growing solid crystallite in the melt. In the former system the inhomogeneity manifests itself best when an electric current is flown through whereas in the latter when the interface expands or contracts as a result of heat current flow. We, however, discuss possibilities of macroscopic current flow as a result of various kind of inhomogeneities in a symmetric periodic potential system. The first case we consider is when $\eta(q)$ and $T(q)$ are space dependent. By taking $\eta(q)$ and $T(q)$ periodic one gets a tilt in the potential field throughout the sample as discussed phenomenologically in ref.[12], resulting in a macroscopic current because of thermal fluctuations. In this case the temperature inhomogeneity is crucial and one can obtain current even when the friction coefficient becomes uniform. Friction coefficient inhomogeneity alone, however, does not generate macroscopic current. In the second case, we consider a thermal particle in a system with space dependent friction coefficient but subjected to external white noise fluctuations, and in the third case a thermal particle subjected to external space dependent white noise is considered. Finally, we discuss the case of a Brownian particle coupled to two thermal baths. It should be noted, however, that in all the cases that we are considering we do not require the potential to be ratchetlike nor do we require the fluctuation forces to be correlated in time to obtain macroscopic current.

In section II we provide a derivation of the macroscopic equation of motion in an inhomogeneous system [15] from a microscopic Hamiltonian of a Brownian particle interacting with a (phonon ) heat bath. We, then, obtain proper Smoluchowski equation from the derived Langevin equation of motion following the prescription of Sancho et al [18]. We use, in Sec.III, 
tion coefficient and nonuniform temperature field to obtain nonzero macroscopic current. In the same section we elaborate three other possible cases of inhomogeneous systems where macroscopic current could be possible. These are shown to be the special cases of a general Maxwell's demon type information engine $[16,17]$. The section IV is devoted to conclusions.

\section{Equation of motion in inhomogeneous systems}

We consider an inhomogeneous system where the inhomogeneity could arise either because of the space dependence of friction coefficient, or the nonuniformity of the temperature field or because of the combined effect of both. The effect of the nonuniformity of temperature or temperature gradient, however, cannot be incorporated as a potential term in the Hamiltonian formalism in sharp contrast to, for instance, the amenability of incorporation of electric field gradient in the Hamiltonian of a charged particle. We, therefore, incorporate the effect of temperature inhomogeneity at the end directly into the equation of motion obtained from the microscopic Hamiltonian suited to take care of the space dependence of the friction coefficient.

\section{IIA. Equation of motion in a space dependent friction field.}

We consider a (subsystem) Brownian particle, of mass $M$, described by a coordinate $Q$ and momentum $P$ moving in a potential field $V(Q)$ of the system and being in contact with a thermal(phonon) bath. The bath oscillators are described by coordinates $q_{\alpha}$, momenta $p_{\alpha}$ and mass $m_{\alpha}$ with characteristic frequencies $\omega_{\alpha}$. We consider the total Hamiltonian

$$
H=\frac{P^{2}}{2 M}+V(Q)+\sum_{\alpha}\left[\frac{p_{\alpha}^{2}}{2 m_{\alpha}}+\frac{m_{\alpha} \omega_{\alpha}^{2}}{2}\left(q_{\alpha}-\lambda_{\alpha} \frac{A(Q)}{m_{\alpha} \omega_{\alpha}^{2}}\right)^{2}\right],
$$

The interaction of the subsystem with the thermal bath is through the linear (in $q$ ) coupling term $\lambda_{\alpha} q_{\alpha} A(Q)$. From (2) one obtains the following equations of motion.

$$
\dot{Q}=\frac{P}{},
$$




$$
\begin{gathered}
\dot{P}=-V^{\prime}(Q)+\sum_{\alpha} \lambda_{\alpha} A^{\prime}(Q)\left[q_{\alpha}-\lambda_{\alpha} \frac{A(Q)}{m_{\alpha} \omega_{\alpha}^{2}}\right], \\
\dot{q}=\frac{p_{\alpha}}{m_{\alpha}} \\
\dot{p}_{\alpha}=-m_{\alpha} \omega_{\alpha}^{2} q_{\alpha}+\lambda_{\alpha} A(Q),
\end{gathered}
$$

where $A^{\prime}(Q)$ is the derivative of $A(Q)$ with respect to $Q$. After solving (3c) and (3d) for $q_{\alpha}$ using the method of Laplace transform and substituting its value in (3b), we obtain

$$
\begin{gathered}
\dot{Q}=\frac{P}{M}, \\
\dot{P}(t)=-V^{\prime}(Q)-\sum_{\alpha} \frac{\lambda_{\alpha}^{2} A^{\prime}(Q)}{m_{\alpha} \omega_{\alpha}^{2}} \int_{0}^{t} d x \cos \omega_{\alpha}\left(t-t^{\prime}\right) A^{\prime}(Q) \frac{P\left(t^{\prime}\right)}{M} \\
+A^{\prime}(Q) \sum_{\alpha} \lambda_{\alpha}\left[x_{\alpha}(0) \cos \left(\omega_{\alpha} t\right)+\frac{\dot{x}_{\alpha}(0)}{\omega_{\alpha}} \sin \left(\omega_{\alpha} t\right)\right]+A^{\prime}(Q) \sum_{\alpha} \frac{A\left(Q_{0}\right) \lambda_{\alpha}^{2}}{m_{\alpha} \omega_{\alpha}^{2}} \cos \left(\omega_{\alpha} t\right) .
\end{gathered}
$$

Here $Q_{0}$ is the initial value of the particle co-ordinate $Q$ and $x_{\alpha}(0)$ and $\dot{x}_{\alpha}(0)$ are the initial co-ordinates and velocities, respectively, of the bath variables. The second term in the right hand side of equation (4b) depends on the momenta at all times previous to $t$. At this stage Markovian limit is imposed so that

$$
g\left(t-t^{\prime}\right)=\sum_{\alpha} \frac{\lambda_{\alpha}^{2}}{m_{\alpha} \omega_{\alpha}^{2}} \cos \omega_{\alpha}\left(t-t^{\prime}\right)=2 \eta \delta\left(t-t^{\prime}\right)
$$

The equation (5) follows readily from the well known Ohmic spectral density distribution for the bath oscillators, i.e.,

$$
\rho(\omega)=\frac{\pi}{2} \sum_{\alpha} \frac{\lambda_{\alpha}^{2}}{m \omega_{\alpha}} \delta\left(\omega-\omega_{\alpha}\right)=\eta \omega e^{-\frac{\omega}{\omega_{c}}} .
$$

where $\omega_{c}$ is an upper cutoff frequency set by the oscillator spectrum of the thermal bath. The Markovian approximation (5) has the effect of neglecting the transient terms involving the 
the equation should well describe the motion of the Brownian particle in time scales $t>\omega_{c}^{-1}$. The equation of motion thus assumes the form

$$
\begin{gathered}
\dot{Q}=\frac{P}{M} \\
\dot{P}=-V^{\prime}(Q)-\frac{\eta}{M}\left[A^{\prime}(Q)\right]^{2} P+A^{\prime}(Q) f(t),
\end{gathered}
$$

where

$$
f(t)=\sum_{\alpha} \lambda_{\alpha}\left[q_{\alpha}(0) \cos \left(\omega_{\alpha} t\right)+\frac{\dot{q}_{\alpha}(0)}{\omega_{\alpha}} \sin \left(\omega_{\alpha} t\right)\right] .
$$

The force $f(t)$ is fluctuating in character because of the associated uncertainties in the initial conditions $q_{\alpha}(0)$ and $\dot{q}_{\alpha}(0)$ of the bath variables. However, as the thermal bath is characterized by its temperature $T$, the equilibrium distribution $P_{e q}\left(q_{\alpha}(0), \dot{q}_{\alpha}(0)\right)$ of bath variables is given by the Boltzmannian form

$$
P\left(q_{\alpha}(0), \dot{q}_{\alpha}(0)\right)=\frac{1}{Z} \prod_{\alpha} e^{-\frac{1}{2 k_{B} T}\left(m_{\alpha} \dot{q}_{\alpha}^{2}(0)+m_{\alpha} \omega_{\alpha}^{2} q_{\alpha}^{2}(0)\right)},
$$

where $Z$ is the partition function. Using equation (8a) and (6) one can easily compute the statistical properties of the fluctuating force $f(t)$. It is Gaussian with

$$
\langle f(t)\rangle=0,
$$

and

$$
\left\langle f(t) f\left(t^{\prime}\right)\right\rangle=k_{B} T g\left(t-t^{\prime}\right)=2 k_{B} T \eta \delta\left(t-t^{\prime}\right) .
$$

It should be noted that the effect of the interaction term $\lambda_{\alpha} q_{\alpha} A(Q)$ in the Hamiltonian (2) is to 
$A^{\prime}(Q)=$ constant corresponds to a uniform friction coefficient. We redefine, $\left[A^{\prime}(Q)\right]^{2} \eta=\eta(Q)$ and $\frac{f(t)}{\sqrt{T}} \rightarrow f(t)$, and put $M=1$, in (7) to obtain,

$$
\begin{gathered}
\dot{Q}=P \\
\dot{P}=-V^{\prime}(Q)-\eta(Q) P+\sqrt{k_{B} T \eta(Q)} f(t),
\end{gathered}
$$

with

$$
\langle f(t)\rangle=0,
$$

and

$$
\left\langle f(t) f\left(t^{\prime}\right)\right\rangle=2 \delta\left(t-t^{\prime}\right) .
$$

From eqs.(9) it follows that the derived Langevin equation of motion (10b) of a Brownian particle, in a system with space dependent friction $\eta(Q)$ but at constant uniform temperature $T$, is internally consistent and obeys fluctuation-dissipation theorem. We now proceed to incorporate the effect of space dependence of temperature, in a thermally nonuniform system, into the Langevin equation of motion by assuming that the Brownian particle comes in contact with a continuous sequence of independent temperature baths as its coordinate $q$ changes in time. (For notational simplicity, we replace the coordinate $Q$ and momenta $P$ by the corresponding lower case letters $q$ and $p$, respectively, reserving $P$ for probability distribution.)

\section{IIB. Equation of motion in a space dependent friction and temperature field}

We consider each space point $q$ of the system to be in equilibrium with a thermal bath characterised by temperature $T(q)$. Also, it should be noted that one could take $\eta(q)$ to be 
to an equation of motion with the constant friction coefficient but with the same statistical character of $f(t)$ (11a-11b) in all $q$ intervals. Let us discretize the system, for the sake of argument, into segments $\Delta q$ around $q$ and represent them by indices $i$. Let us further assume that each segment is connected to an independent thermal bath at temperature $T_{i}$ with corresponding random forces $f_{i}(t)$ so that the equation of motion (10b), in the segment $i$,

will have the last term $\sqrt{k_{B} T_{i} \eta(Q)} f_{i}(t)$. As the two different segments $i$ and $j$ are each coupled to an independent tempearture bath we have $\left\langle f_{i}(t) f_{j}\left(t^{\prime}\right)\right\rangle=2 \delta_{i j} \delta\left(t-t^{\prime}\right)$. Because $f(t)$ is $\delta$ correlated in time, as the particle evolves dynamically the fluctuation force $f_{i}(t)$ experienced by the Brownian particle while in the space segment $i$ at time $t$ will have no memory about the fluctuating force experienced by it at some previous time $t^{\prime}$ while in the space segment $j \neq i$. The space-dependent index $i$ in $f_{i}(t)$, therefore, can be ignored and the equation of motion becomes local in time as well as in space. Therefore, in the continuum limit, the stochastic equations of motion of the Brownian particle, in an inhomogeneous medium with space dependent friction and nonuniform temperature, acquire the simple forms

$$
\begin{gathered}
\dot{q}=p, \\
\dot{p}=-V^{\prime}(q)-\eta(q) p+\sqrt{k_{B} T(q) \eta(q)} f(t),
\end{gathered}
$$

with

$$
\left\langle f(t) f\left(t^{\prime}\right)\right\rangle=2 \delta\left(t-t^{\prime}\right) .
$$

\section{IIC. The Smoluchowski Equation}

From eq.(12b) one can readily write down the Fokker-Planck equation or the Kramers equation for the full probability distribution $P(q, v, t)$. However, in most of the practical 
describe the motion of the Brownian particle. This probability distribution $P(q, t)$ can be obtained in the overdamped limit of the Langevin equation (12b) which is valid on time scales larger than the inverse friction $\eta^{-1}$. In other words in the overdamped case the fast variable, velocity $v$, is eliminated from the equation of motion. In the case of homogeneous systems one simply puts $\dot{p}=0$ in eq.(12b) to obtain the overdamped Langevin equation. However, in case of inhomogeneous systems, the above method of adiabatic elimination of fast variables does not work, and leads to unphysical equilibrium distribution. The proper prescription for the elimination of fast variables has been given in Ref.[18] for systems with space dependent friction. The method retains all terms upto order $\eta^{-1}$ and the resulting overdamped Langevin equation yields physically valid equilibrium distribution. We, therefore, apply the same prescription to obtain the overdamped Langevin equation of motion in an inhomogeneous system with space dependent friction $\eta(q)$ and nonuniform temperature field $T(q)$. We obtain,

$$
\dot{q}=-\frac{V^{\prime}(q)}{\eta(q)}-\frac{k_{B}}{2[\eta(q)]^{2}}\left[T(q) \eta^{\prime}(q)+\eta(q) T^{\prime}(q)\right]+\sqrt{\frac{k_{B} T(q)}{\eta(q)}} f(t),
$$

with

$$
\left\langle f(t) f\left(t^{\prime}\right)\right\rangle=2 \delta\left(t-t^{\prime}\right) .
$$

Using van Kampen Lemma[20] and the Novikov's theorem[21] we obtain the corresponding Fokker-Planck equation as

$$
\frac{\partial P(q, t)}{\partial t}=\frac{\partial}{\partial q} \frac{1}{\eta(q)}\left[\frac{\partial}{\partial q} k_{B} T(q) P(q, t)+V^{\prime}(q) P(q, t)\right] .
$$

Eq.(15) is the Smoluchowski equation for an overdamped Brownian particle moving in an in- 
noted that eq.(15) gives the correct form of diffusion equation instead of either of the two forms mentioned in eqs.(1). It is clear that the temperature and the friction coefficients influence the particle motion in a qualitatively different fashion and they cannot be plugged together to get effective diffusion coefficient to satisfy either of the forms of eq.(1). In the next section we discuss how the system inhomogeneity can help maintain a macroscopic unidirectional current.

\section{Macroscopic motion obtained from inhomogeneous systems}

We consider inhomogeneous systems where the inhomogeneity could be an internal property of the system or it could be imposed externally. As mentioned earlier we consider four cases where macroscopic motion can be obtained.

\section{IIIA. Macroscopic motion in an inhomogeneous system with space dependent}

\section{friction and nonuniform temperature}

When the system is bounded at $q \rightarrow \pm \infty$, i.e., $V \rightarrow \infty$ as $q \rightarrow \pm \infty$, the system attains

steady (stationary) state with zero probability current. In such a situation, we can calculate the steady state probability distribution $P_{s}(q)$, from the Smoluchowski equation (15), by setting the probability current

$$
\frac{1}{\eta(q)}\left[V^{\prime}(q) P(q, t)+\frac{\partial}{\partial q} k_{B} T(q) P(q, t)\right]
$$

equal to zero, as

$$
P_{s}(q)=N e^{-\psi(q)},
$$

where

$$
\psi(q)=\int^{q}\left(\frac{V^{\prime}(x)+k T^{\prime}(x)}{k_{0} T(x)}\right) d x,
$$


and $N$ is a normalization constant. It is very clear from the expression, eq.(18), for $\psi(q)$ that the peaks of $P_{s}(q)$ are determined not by the minima of $V(x)$ alone but are determined as a combined effect with $T(x) . P_{s}(q)$ may even peak at positions which would be quite less likely to be populated in the stationary situations for uniform temperature, $T(x)=T$ condition. In this respect, nonequlibrium situations appear strange but are quite common in biological systems where, for example, otherwise less likely ion channels are, in some situations, found to be more active for ionic transport. Recently such nonequlibrium behaviour in biological systems have been theoretically attributed to the effect of nonequlibrium fluctuations and the process has been termed as kinetic focussing[22]. Moreover, it should be noted that the relative stability of two states of a system with nonuniform temperature field is not determined by the local function $V(q)$ but by the entire pathway through a continuous sequence of intervening states between the two states under comparison. The temperature variation may modify the kinetics of these intervening states drastically and hence their contribution towards the relative stability will be substantial even when they are sparsely populated. It should further be noted that $\psi(q)$ is not determined by $\eta(q)$ as it should be. Moreover, the functional form of $\psi(q)$ is similar to $\int^{q} \frac{v(x)}{D(x)} d x$, of course, in this case $V^{\prime}(q)$ has been augmented by a compensating force $\mathrm{k} T^{\prime}(q) \cdot v(q)=\eta^{-1}(q)\left[V^{\prime}(q)+k T^{\prime}(q)\right]$ is the drift velocity and $\mathrm{D}(\mathrm{q})=\eta^{-1}(q) k_{B} T(q)$ is the effective diffusion coefficient.

So far we have not assigned any functional form to $V(q), T(q)$ and $\eta(q)$. In ref.[12] it is shown that at least in one case the system can generate nonzero probability current, namely, when both $V(q)$ and $D(q)$ are periodic with same periodicity but having a phase difference other than 0 and $\pi$. In our present problem if we assume $V(q), T(q)$ and $\eta(q)$ to be periodic 
functions with periodicity, say, $2 \pi$ then the probability current is given by

$$
J=\frac{1-e^{-\delta}}{\int_{0}^{2 \pi} d y e^{-\psi(y)} \int_{y}^{y+2 \pi} d x \frac{e^{\psi(x)}}{D(x)}},
$$

where $\delta=\psi(q)-\psi(q+2 \pi)$ determines the effective slope of a generalized potential $\psi(q)$ and hence $\delta$ being + or - ve determines the direction of current. It is obvious from the expression for $\delta$ that the phase difference $\phi$, between $V(q)$ and $T(q)$, alone determines the direction of current. The net unidirectional current remains nonzero (finite) except when $\phi$ is an integral mutiple of $\pi$ ( $\phi=n \pi$ corresponds to zero effective slope of the generalized potential). It should further be noted that the variation of $\eta(q)$ does not determine the direction of current but does affect the magnitude of current. A periodic variation of $\eta(q)$ and $V(q)$ but uniform $T(q)$ will yield no unidirectional current. For $V(q)=V_{0}(1-\cos (q))$ and $T(q)=T_{0}(1-\alpha \cos (q-\phi))$,

with $0<\alpha<1$ (for positive temperature) $\delta$ turns out to be $\frac{2 \pi V_{0} \sin \phi}{k_{B} T}\left[\frac{1}{\sqrt{1-\alpha^{2}}}-1\right]$ which is definitely nonzero for $\phi \neq n \pi, n=0, \pm 1, \pm 2, \ldots \ldots$. Thus $\phi$ alone determines the direction of nonzero current $J$. In this case the periodic variation of temperature plays the crucial role and may yield current even when $\eta(q)=\eta_{0}=$ constant. We now consider cases, where $\eta(q)$ plays a decisive role.

\section{IIIB. Macroscopic motion in an inhomogeneous system with space dependent}

\section{friction in the presence of an external parametric white noise}

Unlike the case considered in subsection IIIA, where the overdamped Brownian particle experiences a fixed (in time) local (nonuniform) temperature profile $T(q)$ during its sojourn $q(t)$ for all $t$, we consider, in this subsection, a system with uniform temperature $T(q)=T$ but a spatially varying $\eta(q)$. The Langevin equation of motion is given by

$$
\dot{p}=-V^{\prime}(q)-\eta(q) p+\sqrt{k_{B} T \eta(q)} f(t)
$$


and the corresponding overdamped equation is

$$
\dot{q}=-\frac{V^{\prime}}{\eta(q)}-\frac{k_{B} T \eta^{\prime}(q)}{2[\eta(q)]^{2}}+\sqrt{\frac{k_{B} T}{\eta(q)}} f(t),
$$

with

$$
\langle f(t)\rangle=0
$$

and

$$
\left\langle f(t) f\left(t^{\prime}\right)\right\rangle=2 \delta\left(t-t^{\prime}\right) .
$$

We, now, subject the system to an external parametric additive white noise fluctuating force $\xi(t)$, so that the equation of motion becomes

$$
\dot{q}=-\frac{V^{\prime}}{\eta(q)}-\frac{k_{B} T \eta^{\prime}(q)}{2[\eta(q)]^{2}}+\sqrt{\frac{k_{B} T}{\eta(q)}} f(t)+\xi(t),
$$

with

$$
\begin{gathered}
\langle\xi(t)\rangle=0, \\
\left\langle\xi(t) \xi\left(t^{\prime}\right)\right\rangle=2 \Gamma \delta\left(t-t^{\prime}\right),
\end{gathered}
$$

where $\Gamma$ is the strength of the external white noise $\xi(t)$. We can immediately write down the corresponding Fokker-Planck (Smoluchowski) equation

$$
\frac{\partial P}{\partial t}=\frac{\partial}{\partial q}\left[\left\{\frac{V^{\prime}(q)}{\eta(q)}\right\} P+\left\{\frac{k_{B} T}{\eta(q)}+\Gamma\right\} \frac{\partial P}{\partial q}\right]
$$

For periodic functions $V(q)$ and $\eta(q)$, with periodicity $2 \pi$, one obtains unidirectional current following earlier procedure using equation (23). The resulting expression for current $J$ takes the same functional form as given in eq.(19) where $\psi(q)$ is now given by

$$
\psi(q)=\int^{q} d x \frac{V^{\prime}(x)}{k_{B} T+\Gamma \eta(x)}
$$


and the effective diffusion coefficient

$$
D(q)=\left(k_{B} T+\Gamma \eta(q)\right) / \eta(q)
$$

with

$$
\delta=\psi(q)-\psi(q+2 \pi)
$$

For $\mathrm{V}(\mathrm{q})=V_{0}(1-\cos (q))$ and $\eta(q)=\eta_{0}(1-\alpha \cos (q-\phi)) \delta$ turns out to be equal to

$\frac{2 \pi V_{0} \sin \phi}{\alpha \eta_{0}}\left[\frac{k_{B} T+\eta_{0}}{\sqrt{\left(k_{B} T+\eta_{0}\right)^{2}-\left(\eta_{0} \alpha\right)^{2}}}-1\right]$. As earlier the direction of current is determined by the phase difference $\phi$ between the periodic functions $V(q)$ and $\eta(q)$.

It is important to notice that there is no way one could obtain macroscopic current in the absence of the external white noise $\xi(t)$. This case, however, is similiar in essence to the previous case of nonuniform temperature. In the present situation the overdamped Brownian particle is subjected to an external parametric random noise. The noise being externally imposed, the system always absorbs energy (without the presence of corresponding loss factor) [23]. Also, the overdamped particle moves slowly wherever the friction coefficient $\eta(q)$ is large and the possibility of absorption of energy from the external white noise at those elements $q$ of the system, therefore, is correspondingly large. Thus, the effective temperature $T(q)$ of the system is given by $k_{B} T+\Gamma \eta(q)$, which modulates as $\eta(q)$ varies and hence the macroscopic current results as in the case IIIA.

IIIC. Macroscopic motion in a homogeneous system but subjected to an external parametric space dependent white noise

The overdamped Langevin equation is , 
with $\langle f(t)\rangle=0$ and $\left\langle f(t) f\left(t^{\prime}\right)\right\rangle=2 \delta\left(t-t^{\prime}\right)$. Eq.(26) obeys fluctuation-dissipation theorem and hence in the absence of any external bias potential there can be no net current irrespective of the form of the periodic potential $V(q)$. We now subject the system to an external multiplicative Gaussian white noise fluctuation. The corresponding overdamped Langevin equation is given by

$$
\dot{q}=-\frac{V^{\prime}(q)}{\eta}+\sqrt{\frac{k_{B} T}{\eta}} f(t)+g(q) \xi(t)
$$

where $g(q)$ is an arbitrary function of $q$ and $\xi(t)$ is a white noise with

$$
\langle\xi(t)\rangle=0
$$

and

$$
\left\langle\xi(t) \xi\left(t^{\prime}\right)\right\rangle=2 \Gamma \delta\left(t-t^{\prime}\right)
$$

The associated Fokker-Planck equation can be immediately written down as

$$
\frac{\partial P}{\partial t}=\frac{\partial}{\partial q} \frac{V^{\prime}(q)}{\eta} P+\frac{k_{B} T}{\eta} \frac{\partial}{\partial q^{2}} P+\Gamma \frac{\partial}{\partial q} g(q) \frac{\partial}{\partial q} g(q) P
$$

Now, if we assume $V(q)$ and $g(q)$ to be periodic functions with periodicity $2 \pi$, the net unidirectional current can be obtained and is given by eq.(19), with

$$
\psi(q)=\int^{q} d x \frac{V^{\prime}(x)+\eta \Gamma g(x) g^{\prime}(x)}{k_{B} T+\eta \Gamma g^{2}(x)},
$$

and the effective difussion coefficient,

$$
D(q)=\frac{k_{B} T+\eta \Gamma g^{2}(q)}{\eta}
$$

For the specific form of the periodic functions 
and

$$
g(q)=\sqrt{g_{0}(1-\alpha \cos (q-\phi))}
$$

we obtain,

$$
\delta=\frac{2 \pi V_{0} \sin \phi}{\eta \Gamma g_{0} \alpha}\left[\frac{k_{B} T+\eta \Gamma g_{0}}{\sqrt{\left(k_{B} T+\eta \Gamma g_{0}\right)^{2}-\left(\eta \Gamma g_{0}\right)^{2}}}-1\right]
$$

The phase $\phi$ being +ve or -ve determines the sign of $\delta$ and consequently direction of the current $J$ (eq.(19)).

It should be noted that, as in case IIIB, the overdamped Brownian particle experiences an effective space dependent temperature $T(q)=k_{B} T+\eta \Gamma[g(q)]^{2}$. The first case (IIIA) corresponds to a system which is intrinsically nonequilibrium and requires an internal mechanism such as the generation of latent heat at the interface in first order transitions to maintain the temperature profile $T(q)$. The other two cases (IIIB and IIIC) are, however, supplied with energy externally via the externally applied white noise. And finally, we consider a case where the Brownian particle is subjected to two thermal baths.

IIID. Macroscopic motion in an inhomogeneous system under the action of two thermal (noise) baths

We now consider the situation in which the system is in contact with an additive thermal noise bath at temperature $T$ and a multiplicative thermal noise bath at temperature $\bar{T}$. The corresponding equation of motion of the Brownian particle can be derived from a microscopic Hamiltonian and is given by $[16]$

$$
M \ddot{q}=-V^{\prime}(q)-\Gamma(q) \dot{q}+\xi_{A}(t)+\sqrt{f(q)} \xi_{B}(t),
$$

$\xi_{A}(t)$ and $\xi_{B}(t)$ are two independent Gaussian white noise fluctuating forces with statistics, 


$$
\left\langle\xi_{A}(t) \xi_{A}\left(t^{\prime}\right)\right\rangle=2 \Gamma_{A} k_{B} T \delta\left(t-t^{\prime}\right),
$$

and

$$
\begin{gathered}
\left\langle\xi_{B}(t)\right\rangle=0, \\
\left\langle\xi_{B}(t) \xi_{B}\left(t^{\prime}\right)\right\rangle=2 \Gamma_{B} k_{B} \bar{T} \delta\left(t-t^{\prime}\right),
\end{gathered}
$$

where, $T$ and $\bar{T}$ are temperatures of the two baths $\mathrm{A}$ and $\mathrm{B}$, respectively. It should be noted that, $\xi_{A}(t)$ and $\xi_{B}(t)$ represent internal fluctuations and together satisfy the fluctuationdissipation theorem $\Gamma(q)=\Gamma_{A}+\Gamma_{B} f(q)$. The bath B is associated with a space dependent friction coefficient $f(q)$. When the two temperatures $T$ and $\bar{T}$ become equal the system will be in equilibrium and no net current can flow. By making $T$ and $\bar{T}$ different the system is rendered nonequilibrium and one can extract energy at the expense of increased entropy. The system, thus, acts as a Maxwell's-demon type information engine which extracts work by rectifying internal fluctuations. In ref. [16] an expression for current is obtained in the overdamped limit. The overdamped limit of the Langevin equation is taken by setting the left hand side of eq.(30) equal to zero. This procedure of obtaining overdamped limit is not correct as explained in section I. Following the procedure of ref.[18] the correct Fokker-Planck equation in the overdamped limit is given by [17]

$$
\begin{aligned}
\frac{\partial P}{\partial t}=\frac{\partial}{\partial q}\left\{\frac{V^{\prime}(q)}{\Gamma(q)} P+\frac{T \Gamma_{A}}{\Gamma(q)} \frac{\partial}{\partial q} \frac{P}{\Gamma(q)}\right. & +\bar{T} \Gamma_{B} \frac{\sqrt{f(q)}}{\Gamma(q)} \frac{\partial}{\partial q} \frac{\sqrt{f(q)}}{\Gamma(q)} P \\
& \left.+\bar{T} \Gamma_{B} \frac{(\sqrt{f(q)})^{\prime} \sqrt{f(q)}}{[\Gamma(q)]^{2}} P\right\} .
\end{aligned}
$$

For periodic functions $V(q)$ and $f(q)$ with periodicity $2 \pi$ the noise induced transport current $J$ is given by eq.(19), where, now 
and $D(q)=\frac{T \Gamma_{A}+\bar{T} \Gamma_{B} f(q)}{(\Gamma(q))^{2}}$ and $\delta=\psi(q)-\psi(q+2 \pi)$.

As in earlier cases, taking specific periodic forms of $V(q)=V_{0}(1-\cos q)$ and $f(q)=$ $f_{0}(1-\alpha \cos (q-\phi))$, the exponent $\delta$ in eq.(19) for current is obtained as

$$
\delta=\left(1-\frac{T}{\bar{T}}\right) \frac{2 \pi V_{0} \sin \phi}{\bar{T} \Gamma_{B} f_{0} \alpha}\left[\frac{T \Gamma_{A}+\bar{T} \Gamma_{B} f_{0}}{\sqrt{\left(T \Gamma_{A}+\bar{T} \Gamma_{B} f_{0}\right)^{2}-\left(\bar{T} \Gamma_{B} f_{0} \alpha\right)^{2}}}-1\right]
$$

It is clear from the expression for $\delta$ that, again as in earlier cases (IIIA-C), the phase difference $\phi$ between $V(q)$ and $f(q)$ determines the direction of current $J$. It is to be noted that, the current will flow in one direction if $T>\bar{T}$ and will flow in the opposite if $T<\bar{T}$ for given $\phi$. Thus, the system acts like a Carnot engine which extracts work by making use of two thermal baths at different temperatures $(T \neq \bar{T})$. Moreover $\delta$ vanishes when $f(q)$ becomes space independent constant $f_{0}$, i.e., when $\alpha=0$, and the current $J$ becomes zero. It should be noted further that when the amplitudes of $f(q)$ and $f^{\prime}(q)$ are small compared to the amplitude of $V(q)$, the problem turns out to be equivalent to a particle moving in a spatially varying temperature field, $T(q)=\left(T \Gamma_{A}+\bar{T} \Gamma_{B} f(q)\right) / \Gamma(q)$ and, as discussed in section IIIA, such a nonuniform temperature field yields net unidirectional current.

\section{Summary and Discusssion}

Transport in a nonequlibrium periodic system has become, in recent times, a field of very active research. We have just tried, in the beginning of this work, only to enumerate various working ideas to build a plausible model of thermal ratchet. The brief enumeration is, of course, not complete. The models are being gradually refined and simplified to be close either to the experimental reality or to invent techniques to be useful in practice. For example, there are attempts to show that one can obtain macroscopic current in a symmetric periodic potential system with the application of (1) zero averaged external white shot noise 
asymmetric fluctuations [10], or, (3) zero averaged asymmetric noise form with one large kick in one direction and two smaller kicks of half the strength of the former in the opposite direction [25]. Moreover, it has further been reported to obtain macroscopic motion with the use of two baths one thermal other athermal (time correlated) in a ratchetlike potential system [26]. In these situations even current reversal is possible. It has further been reported that with the application of suitable voltage fluctuations a voltage-sensitive macromolecule can be put into a desired (but otherwise energetically unfavourable) kinetic substate [22]. These vast new developements in the study of transport phenomena could be but interesting variants, in principle, of a simple unified theoretical framework of inhomogeneous nonequlibrium systems. Transport in inhomogeneous nonequilibrium systems has attracted attention since long. We have presented in Sec.IIIA a microscopic approach to obtain macroscopic equation of motion in such systems.

In the situations considered, in sections IIIB and IIIC, the system is subjected to external fluctuations violating the fluctuation- dissipation theorem. Also, these two cases, in a sense, are physically equivalent to having a spatially varying temperature field as considered in section IIIA. However, in the situation IIID both the temperature baths were internal parts of the system and the system is subjected to a space dependent friction at temperature $\bar{T}$. Also in the limit of small friction field modulation amplitude the situation corresponds to a spatially varying temperature field. These observations seem to suggest that the case of inhomogeneous systems with spatially varying temperature field provides a general paradigm to obtain macroscopic current and several variants considered to model fluctuation induced transport may fall in the same general class of problems as considered in section IIIA. For example, earlier models of thermal ratchets driven by colored noise in a small correlation time 
to a Brownian particle moving in an inhomogeneous medium with space dependent diffusion coefficient [27]. The interesting idea of relative stability of states, which affects current, in the presence of temperature nonuniformity, however, has not received the attention it deserves.

To conclude, we remark that we do not require the periodic potential field of the system to be ratchetlike nor do we require the fluctuating force to be a colored noise to obtain macroscopic current. Instead, we put emphasis on the role of inhomogeneity of the system to obtain unidirectional current. These inhomogeneities by themselves (section III A) or with the help of external white noise (section III B and III C) or with two temperature baths at different temperatures, one of the bath being athermal (section IIID), satisfy all the conditions to obtain the macroscopic current. To summarise, in the present work, we have given a microscopic basis to obtain unidirectional current in an inhomogeneous system. The present work may help to put the problem of macroscopic unidirectional motion in nonequilibrium systems on a more general footing. 


\section{References}

[1] M. Magnasco, Phys. Rev. Lett. 71, 1477(1993).

[2] R. Bartussek, P. Hänggi, and J. G. Kissner, Europhys. Lett. 28 , 459 (1994).

[3] R. D. Astumian and M. Bier, Phys. Rev. Lett. 72, 1776(1994).

[4] C. R. Doering, W. Horsethemke and J. Riordan, Phys. Rev. Lett. 72, 2984(1994)

[5] A. Mielke, Ann. Physik 4, 476(1995).

[6] M. M. Millonas and M. I. Dykman, Phys. Lett. A185, 65(1994).

[7] J. Prost, J. F. Chauwin, L. Peliti and A. Ajdari, Phys. Rev. Lett. 72, 2652(1994); J. Rousselet, L. Salome, A. Ajdari and J. Prost, Nature 370, 446(1994); J. F. Chauwin, A. Ajdari and J. Prost, Europhys. Lett. 32, 373(1995).

[8] A. Ajdari, D. Mukamel, L. Peliti and J. Prost, J. Phys.(paris) I4, 1551(1994).

[9] M. C. Mahato and A. M. Jayannavar, Phys. Lett. A209, 21(1995).

[10] D. R. Chialvo and M. M. Millonas Phys. Lett A209, 26(1995).

[11] K. Svoboda, C. F. Schmitt, B. J. Schnapp and S. M. Block, Nature , 365, 721(1993).

[12] M. Büttiker, Z. Phys. B - Condensed Matter 68 , 161(1987).

[13] N. G. van Kampen, J. Math. Phys. 29, 1220(1988); Z. Phys. B 68, 135(1987); IBM J. Res. Develop. 32, 107(1988).

[14] R. Landauer, Phys. Today, November 1978, pp.23; Physica A194 , 551(1993); J. Stat. 
[15] A. M. Jayannavar and M. C. Mahato, Pramana - J. Phys. 45, 369(1995).

[16] M. M. Millonas, Phys. Rev. Lett. 74, 10(1995).

[17] A. M. Jayannavar, Phys. Rev. E 53, 2957(1996).

[18] J. M. Sancho, M. San Miguel and D. Dürr, J. Stat. Phys. 28, 291(1982).

[19] A. M. Jayannavar, Z. Phys. B 82, 153(1991).

[20] N. G. van Kampen, Phys. Rep. C24, 172(1976).

[21] E. A. Novikov, Zh. Eksp. Teor. Fiz. 47, 1919(1964); Sov. Phys. JETP 20, 1290(1965).

[22] M. M. Millonas and D. R. Chialvo, Phys. Rev. Lett. 76, 550(1996).

[23] A. M. Jayannavar and N. Kumar, Phys. Rev. Lett. 48, 553(1982); A. M. Jayannavar, Phys. Rev. E48, 837(1993).

[24] J. Luczka, R. Bartussek, and P. Hänggi, Europhys. Lett. 31, 431(1995).

[25] R. Lahiri, IISc preprint (1995).

[26] R. Bartussek, P. Reimann, and P. Hänggi, Phys. Rev. Lett., 76, 1166(1996).

[27] A. J. R. Madurveira, P. Hänggi, V. Buonamano, and W. A. Rodriguez, Phys. Rev. E 51, 3849 (1995); R. Bartussek, A. J. R. Madureira, and P. Hänggi, ibid. 52, 2149(1995). 